\title{
Total Sleep Deprivation Decreases Immobility in the Forced-Swim Test
}

\author{
Faustino Lopez-Rodriguez ${ }^{*, 1,2}$, Joseph Kim' and Russell E Poland ${ }^{\mathbf{2 , 3}}$ \\ 'Department of Psychiatry and Biobehavioral Sciences, UCLA School of Medicine, Los Angeles, CA, USA; ${ }^{2}$ Brain Research Institute, UCLA School \\ of Medicine, Los Angeles, CA, USA; ${ }^{3}$ Department of Psychiatry, Cedars Sinai Medical Center, Los Angeles, CA, USA
}

\begin{abstract}
Sleep deprivation can exert antidepressant effects in humans in less than $24 \mathrm{~h}$, making it the fastest acting antidepressant treatment. However, it is rarely used clinically because the effect disappears once the subject goes back to sleep. An understanding of the neurobiological mechanisms underlying the antidepressant effect of sleep deprivation should help to develop new rapidly acting antidepressant strategies. In the present report, an animal model of depression (the forced-swim test) was used to determine whether the effects of total sleep deprivation parallel those obtained with antidepressant drugs. Using the disk-over-water method, rats deprived of sleep for $24 \mathrm{~h}$ exhibited increased swimming behavior when compared to cage control rats, mimicking the effects of serotonergic antidepressants. After $48 \mathrm{~h}$, sleep-deprived rats exhibited increased swimming when compared to both cage control and stimulus control rats, demonstrating that the effect is due to sleep deprivation per se, and not to extraneous factors inherent in the sleep deprivation protocol (such as stress and movement). We believe that this paradigm can be used to study the neurobiological mechanisms of rapid antidepressant effects induced by sleep deprivation.

Neuropsychopharmacology (2004) 29, I I05-I I I I, advance online publication, I 8 February 2004; doi: I 0. I 038/sj.npp. I 300406
\end{abstract}

Keywords: sleep deprivation; depression; animal models; corticosterone

\section{INTRODUCTION}

Antidepressant drugs need to be administered for several weeks before a therapeutic response is achieved (Artigas et al, 1996). However, total sleep deprivation produces antidepressant effects after only one night of deprivation (Gerner et al, 1979). Consequently, total sleep deprivation must induce neurobiological changes that result in rapid improvement of symptoms of depression. An understanding of these changes will help to develop alternative pharmacological strategies that are faster than the ones currently available.

In addition to antidepressant properties, sleep deprivation induces other behavioral effects, such as sedation and a decrease in attention and concentration (Post et al, 1976; Gerner et al, 1979; Pilcher and Huffcutt, 1996). It is thus of paramount importance to determine the relationships between a particular neurobiological change induced by sleep deprivation and the behavioral effect under study. For example, we have previously shown that total sleep deprivation induces an increase in extracellular levels of

*Correspondence: Dr F Lopez-Rodriguez, Neuropsychiatry Institute and Hospital, 37-443, David Geffen UCLA School of Medicine, 740 Westwood Plaza, Los Angeles, CA 90024, USA, Tel: + I 310206 7|44, Fax: + I 310825 7642, E-mail: Faustino@ucla.edu

Received 26 June 2003; revised 03 November 2003; accepted 29 December 2003

Online publication: 9 January 2004 at http://www.acnp.org/citations/ Npp 109040328I/default.pdf serotonin (5-hydroxy-tryptamine, 5-HT) in the posterior hippocampus of the rat (Lopez-Rodriguez et al, 2003), but it still remains to be determined whether this $5 \mathrm{HT}$ increase is related to the antidepressant effects of sleep deprivation. In this regard, we first need to ascertain whether total sleep deprivation induces behavioral effects in rats that parallel those produced by antidepressants. This would allow us to use total sleep deprivation as a tool to study the mechanisms of action of a rapidly acting antidepressant.

Neurobiological studies of sleep deprivation that require invasive procedures are facilitated by the development of animal models. Most of the basic research studies that have addressed the behavioral effects of sleep deprivation use the platform technique, which consists of placing a rat on a $6 \mathrm{~cm}$ platform surrounded by water. This model is limited to deprivation of rapid eye movement (REM) sleep. Although REM sleep deprivation also exerts antidepressant effects in humans, it suffers from two important limitations. First, it takes longer to produce an antidepressant effect (Vogel, 1975). For example, $48 \mathrm{~h}$ of REM sleep deprivation in elderly depressed patients did not have an effect on mood ratings (Reynolds et al, 1990). Second, REM sleep deprivation is more difficult to perform, in that patients have to be connected to an electroencephalogram and a trained technician has to be present constantly to awaken patients when this state is detected (Vogel, 1975). Consequently, less is known about neurobiological changes in humans after REM sleep deprivation since there are fewer studies 
addressing this issue. Owing to the limitations inherent to REM sleep deprivation, we believe that animal models of total sleep deprivation will contribute most to the understanding of rapid antidepressant effects, and will allow more comparisons with human studies.

On the other hand, behavioral effects of total sleep deprivation in animals have not been extensively addressed. In one study, rats were sleep deprived by placing them in a slowly rotating cylinder, an intervention that reversed the effects of social defeat on open field behavior (Meerlo et al, 1996). However, this particular method of total sleep deprivation lacks a control for the physical effect and the stress induced by the movement of the cylinder. In order to control for these extraneous effects, Rechtschaffen and Bergmann (1995) developed a method called the disk-overwater method. Briefly, this method consists of housing two rats (sleep deprived and stimulus control), each in a clear plastic cage with a common central floor in the form of a disk that is surrounded by water. When the sleep-deprived rat falls asleep, the disk rotates for $2 \mathrm{~s}$. In turn, both rats have to walk in an opposite direction to the movement of the disk. When the stimulus control rat falls asleep, the disk does not move, thus allowing the control rat to sleep as long as the sleep-deprived rat is awake (see Method section for more details). Because of these advantages, we chose to use the disk-over-water method to study the antidepressant effects of total sleep deprivation.

There are several models of depression in animals (Willner, 1990; Cryan et al, 2002). One of the models that has been extensively used is the forced-swim test because of its predictive validity and the relative simplicity of its use (Porsolt et al, 1978; Borsini and Meli, 1988). This method consists of placing a rat or mouse in a tank of water. After attempts on the part of the rat to escape and swim in the tank, the animal will eventually become immobile and float. Antidepressants drugs, REM sleep deprivation and electroconvulsive therapy decrease immobility (Porsolt et al, 1977, 1978; Hawkins et al, 1980; van Luijtelaar and Coenen, 1985; Detke et al, 1995; Cryan et al, 2002). It remains to be determined whether total sleep deprivation also decreases immobility in the forced-swim test and, if it does, what is the duration needed to produce such an effect.

In the present study, we determine the effect of total sleep deprivation using the disk-over-water method on the forcedswim test. Our hypothesis was that total sleep deprivation would decrease immobility after $24 \mathrm{~h}$ of deprivation.

\section{METHODS}

Experiments were performed in Sprague-Dawley rats, weighing 330-440 g. Animals $(n=24)$ were kept on a $12 \mathrm{~h}$ light/dark cycle, with lights on at 0600 . Food and water were available ad libitum. Animal procedures followed the guidelines of the Animal Welfare Act, and were approved by the Animal Research Committee of the University of California, Los Angeles. All efforts were made to minimize the number of animals used and their suffering.

\section{Surgery}

Surgery was performed using an anesthesia combination of ketamine $\mathrm{HCl}$ (100 mg/kg i.m.), xylazine (4 mg/kg i.m.), and acepromazine $(0.75 \mathrm{mg} / \mathrm{kg}$ i.m.). Flunixien meglumine $(0.5 \mathrm{mg} / \mathrm{kg}$ i.m. $)$ was used for additional postoperative analgesia (every $12 \mathrm{~h}$ for $48 \mathrm{~h}$ ). Animals were implanted with stainless-steel screw electrodes for lateral (bregma coordinates $\mathrm{AP}=-1.0, \mathrm{~L}=3.0)$ and medial $(\mathrm{AP}=$ midpoint between bregma and lambda, $\mathrm{L}=1.0$ ) cortical electroencephalogram (EEG) recording. Teflon-coated stainless-steel wires, stripped of the insulation in the last $3 \mathrm{~mm}$, were placed in the neck muscle for electromyogram (EMG) recording.

\section{Electrophysiological Recording}

At 1 week after the surgery, animals were moved to the experimental cage and connected through cables to a 12channel commutator to allow recordings in a freely moving condition. Three four-channel MOSFET input operational amplifiers, mounted in the cable connector, served to eliminate cable movement artifacts. Electrophysiological data were amplified and filtered (band pass: $0.3-100 \mathrm{~Hz}$ for cortical recordings and $10-3000 \mathrm{~Hz}$ for EMG recordings), digitized at $500 \mathrm{~Hz} / \mathrm{channel}$ and stored in computer media.

\section{Staging and Measures of Sleep-Wake Cycles}

Using an automatic detection system, behavioral states were scored in epochs of $15 \mathrm{~s}$ into four stages: (1) waking: cortical desynchronization, and medium-high EMG; (2) non-REM sleep: cortical and hippocampal high voltage slow waves and low amplitude EMG; (3) REM sleep: desynchronized cortical EEG, regular pattern of hippocampal theta activity, and very low amplitude EMG.

In addition to studying the total duration of each state over the course of the $48 \mathrm{~h}$ of the experiment, we measured the duration of individual sleep bouts in order to determine whether sleep bouts were different in the sleep deprived $v s$ the control animals. It has been shown that sleep fragmentation leads to increased objective sleepiness and decreased psychomotor performance, even when total sleep was normal (Bonnet and Arand, 2003). In order to perform this analysis, we averaged the duration of sleep bouts in each rat. Separate analyses were performed for non-REM and REM sleep bouts.

\section{Sleep Deprivation Procedure}

Rats were sleep deprived using the disk-over-water method, which is described in detail elsewhere (Rechtschaffen et al, 1983). Briefly, this method consists of housing two rats, one in each of two clear plastic cages, sharing a floor made up of a smooth moveable plastic disk with its center in an alley between the cages. Beneath the disk is a metal tray, which is filled with water $2-3 \mathrm{~cm}$ deep. Each rat is connected by a flexible cable to a commutator to allow for electrophysiological recordings in a freely moving fashion, as explained above. Onset of non-REM sleep in the rat to be sleep deprived is automatically detected when average EMG during a $2 \mathrm{~s}$ epoch falls below a preset value, and the lateral cortex EEG delta power rises above another preset value (these values are preset by analyzing the recordings of each rat during spontaneous wakefulness and non-REM sleep episodes previously recorded). When criteria for non-REM 
sleep are reached in the sleep-deprived rat, the disk rotates until these criteria are no longer present in a $2 \mathrm{~s}$ epoch. When the disk moves, both rats have to walk in a direction opposite to the rotation of the disk to avoid being carried into the water. Sleeping in the stimulus control rats did not trigger movement of the disk. Therefore, the stimulus control animals had opportunity to sleep during those times that the sleep-deprived rats were awake. In all, $300 \mathrm{~W}$ red lamps were placed at a distance of $30 \mathrm{~cm}$ of each cage to warm the animals.

\section{Forced-Swim Test}

Rats are placed individually in a tank $(50 \mathrm{~cm}$ height, $30 \mathrm{~cm}$ diameter) filled with water to a depth of $25 \mathrm{~cm}$, at $25^{\circ} \mathrm{C}$, for $15 \mathrm{~min}$. After each test, rats were dried with a towel and placed in a separate cage, heated by a $300 \mathrm{~W}$ lamp until the animals were dry. Rats were videotaped during the test for further scoring. Behavioral scoring was performed as described by Detke et al (1995). Immobility was defined as the behavior consisting of floating or movement of hind limbs directed exclusively to maintain the head off the water. Climbing was defined as forceful attempts to climb the wall of the tank with either forepaws, or trying to jump out of the tank. Swimming was defined as an active behavior, other than movements characterized as climbing, and which involved displacement around the surface of the water. The behavioral test was scored by a single observer, blind to the treatment condition. Quantification of behaviors was performed by recording the predominant behavior in each $5 \mathrm{~s}$ period (Cryan and Lucki, 2000). We validated the scoring of the forced-swim test in our laboratory by treating additional normal control rats with desipramine $(20 \mathrm{mg} / \mathrm{kg}$ subcutaneous) following standard procedures (Page et al, 1999). Results were similar to those reported previously: that is, increased climbing activity $(17.8 \pm 2.7$ counts $v s 6.0 \pm 3.8$ in saline treated animals $)$ and decreased immobility $(24.0 \pm 2.3$ vs $38.3 \pm 6.6$ in saline animals (Page et al, 1999).

\section{Corticosterone Assay}

Serum corticosterone was analyzed by radioimmunoassay, as previously described (Poland et al, 1994). Briefly, corticosterone was measured using anticorticosterone antisera and I-125 corticosterone obtained form ICN Biomedicals. Sensitivity of the assay was $0.25 \mathrm{ng} / \mathrm{ml}$. As determined by multiple plasma pool replicates, intra-assay variability was $<7 \%$.

\section{Experimental Paradigm}

There were three groups of rats: sleep deprived, stimulus control and cage control. Sleep-deprived rats were submitted to a sleep deprivation protocol as described above. The stimulus control rats received the same environmental stimulation as the sleep-deprived rats, but were allowed to sleep. A third group (cage control) consisted of rats placed in pairs in the disk-over-water apparatus (one in each cage), but without any movement of the disk, and with bedding instead of water. All rats were placed in the disk-over-water apparatus 4 days before the beginning of the recordings for adaptation. The forced-swim test was performed between 1400 and 1500 each day for 3 consecutive days. This test was performed one time before the beginning of the experiments (training session), as well as 24 and $48 \mathrm{~h}$ after sleep deprivation. The sleep deprivation and stimulus control paradigm began at 1700 . The order in which the forcedswim test was performed alternated from day-to-day between the two rats placed in the disk-over-water apparatus. Rats were killed with $\mathrm{CO}_{2}$ and decapitated $48 \mathrm{~h}$ after the beginning of the sleep-deprivation paradigm. This was $25 \mathrm{~min}$ after the beginning of the last forced-swim test, or at equivalent times in the cage control rats. In tota, $1 \mathrm{ml}$ of blood from the decapitation incision was collected in a centrifuge tube containing $50 \mu \mathrm{l}$ of EDTA, shaken, and centrifuged at 15000 r.p.m. for $5 \mathrm{~min}$. In total, $100 \mu$ of the supernatant was collected into a vial, and frozen at $-80^{\circ} \mathrm{C}$ until analyzed for corticosterone. Plasma samples from two sleep-deprived rats and two stimulus control rats were not obtained. Suprarenal glands were dissected and weighted.

\section{Statistical Analysis}

The amount of each stage of the sleep-wake cycle as well as average sleep bout durations were analyzed using one-way ANOVA, with group of rat being the treatment factor, and amount of each of the sleep states as the dependent variables. Weight, age, weight difference, suprarenal weight and serum corticosterone were also analyzed using one-way ANOVA, in which experimental group was the betweensubjects factor.

Behavior was analyzed using two-way repeated measures ANOVA, in which the day was the within-subjects factor, and experimental group the between-subjects factor. Separate ANOVAs were performed for each of the behaviors studied. Fisher's least-square distance was used for post hoc analysis.

\section{RESULTS}

A total of 24 rats were used for the present study, eight per group.

\section{Sleep}

The total amount of wakefulness $\left(\mathrm{F}_{2,15}=83.3, p<0.005\right)$, non-REM sleep $\left(\mathrm{F}_{2,15}=56.9, p<0.005\right)$, and REM sleep $\left(\mathrm{F}_{2,15}=31.1, \quad p<0.005\right) \quad$ were different among groups (Table 1). Amount of wakefulness was higher in sleepdeprived animals, as compared to both the stimulus control and the cage control. Stimulus control rats also had a higher amount of wakefulness than cage control rats. Non-REM sleep was reduced in sleep-deprived rats compared to both control groups, and stimulus control rats displayed less non-REM sleep than cage control rats. REM sleep was not significantly different between sleep-deprived and stimulus control groups, but both groups displayed less REM than cage control group.

When the 2 days of the experiment were compared separately, the amount of wakefulness (day $1: \mathrm{F}_{2,15}=87.6$, $p<0.005$; day $2: \mathrm{F}=50.6, p<0.005)$ and non-REM sleep (day 1: $\mathrm{F}_{2,15}=54.3, p<0.005$; day $2: \mathrm{F}_{2,15}=37.1, p<0.005$ ) was different among all groups. REM sleep (day 1: 
Table I Amount of Sleep and Wakefulness in the Three Groups, Expressed as Percentage Spent in Each State

\begin{tabular}{lccc}
\hline & Day I & Day 2 & Total \\
\hline Sleep deprived & & & \\
W & $95.7 \pm 1.9^{\mathrm{a}, \mathrm{b}}$ & $92.9 \pm 1.8^{\mathrm{a}, \mathrm{b}}$ & $93.5 \pm 2.0^{\mathrm{a}, \mathrm{b}}$ \\
Non-REM & $4.3 \pm 1.9^{\mathrm{a}, \mathrm{b}}$ & $6.5 \pm 1.3^{\mathrm{a}, \mathrm{b}}$ & $5.9 \pm 1.6^{\mathrm{a}, \mathrm{b}}$ \\
REM & $0.1 \pm 0^{\mathrm{b}}$ & $0.6 \pm 0.5^{\mathrm{b}}$ & $0.6 \pm 0.5^{\mathrm{b}}$ \\
& & & \\
Stimulus control & & & \\
W & $85.0 \pm 2.0^{\mathrm{b}}$ & $73.2 \pm 5.1^{\mathrm{b}}$ & $79.1 \pm 3.1^{\mathrm{b}}$ \\
Non-REM & $14.3 \pm 1.8^{\mathrm{b}}$ & $25.0 \pm 5.0^{\mathrm{b}}$ & $19.7 \pm 3.1^{\mathrm{b}}$ \\
REM & $0.7 \pm 0.5^{\mathrm{b}}$ & $1.7 \pm 0.3^{\mathrm{b}}$ & $1.2 \pm 0.4^{\mathrm{b}}$ \\
& & & \\
Cage control & & & $50.1 \pm 3.0$ \\
W & $49.5 \pm 4.2$ & $50.9 \pm 3.2$ & $42.4 \pm 3.5$ \\
Non-REM & $42.1 \pm 4.5$ & $42.1 \pm 3.5$ & $7.5 \pm 1.1^{-1}$ \\
REM & $8.3 \pm 1.8$ & $7.0 \pm 0.7$ & \\
\hline
\end{tabular}

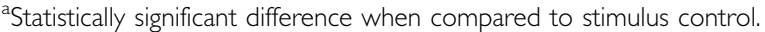

bStatistically significant difference when compare to cage control.

$\mathrm{F}_{2,15}=22.9, p<0.005$; day $\left.2: \mathrm{F}_{2,15}=42.8, p<0.005\right)$ was significantly greater in cage control group as compared to the sleep-deprived group or the stimulus control group. However, the difference between sleep-deprived and stimulus control groups did not reach statistical significance.

Average sleep bout durations of non-REM sleep $\left(\mathrm{F}_{2,15}=26.5, \quad p<0.0001\right)$ and $\mathrm{REM}$ sleep $\left(\mathrm{F}_{2,15}=29.5\right.$, $p<0.0001$ ) were significantly different among the three groups. Post hoc analysis demonstrated a difference in all pairwise comparisons, in which the duration of each state was compared with duration of the same state in the other two groups of rats (in each day). Thus, though there was no statistically significant difference between total duration of REM sleep between sleep-deprived and stimulus control rats, the average duration of individual REM sleep episodes was higher in stimulus control rats than in sleep-deprived rats (Table 2).

\section{Behavior}

Immobility was different among the groups after 24 $\left(\mathrm{F}_{2,21}=5.48, \quad p<0.05\right)$ and $48 \mathrm{~h} \quad\left(\mathrm{~F}_{2,21}=6.79, \quad p<0.01\right.$; Figure 1). After $24 \mathrm{~h}$, immobility in the sleep-deprived and stimulus control rats was not different, but both groups displayed less immobility than cage control rats. After $48 \mathrm{~h}$, sleep-deprived rats displayed less immobility than stimulus control and cage control rats, and there was no difference between the two control groups (Figure 1).

Swimming behavior also was different among groups after $24 \quad\left(\mathrm{~F}_{2,21}=5.46, p<0.05\right)$ and $48 \mathrm{~h} \quad\left(\mathrm{~F}_{2,21}=7.13\right.$, $p<0.01)$. After $24 \mathrm{~h}$, sleep-deprived and stimulus control rats did not differ in the amount of swimming, but both groups swam for more time than cage control rats. After $48 \mathrm{~h}$, sleep-deprived rats swam more than stimulus control and cage control rats, but there was no difference in the amount of swimming between the two control groups (Figure 1).
Table 2 Average Duration of Non-REM and REM Sleep Episodes in Each of the Three Groups

\begin{tabular}{lccc}
\hline & \multicolumn{3}{c}{ Duration (s) } \\
\cline { 2 - 4 } & Day I & Day 2 & Total \\
\hline Sleep deprived & & \\
Non-REM & $18.4 \pm 4.0$ & $19.9 \pm 1.3$ & $19.1 \pm 2.5$ \\
REM & $6.4 \pm 3.3$ & $21.4 \pm 10.5$ & $13.9 \pm 4.8$ \\
& & & \\
Stimulus control & & & $39.2 \pm 3.7$ \\
Non-REM & $36.7 \pm 3.0$ & $37.9 \pm 4.9$ & $29.3 \pm 2.8$ \\
REM & $20.8 \pm 4.9$ & & $74.2 \pm 9.9$ \\
Cage control & & & $51.1 \pm 2.7$ \\
Non-REM & & & \\
REM & $71.9 \pm 9.6$ & $50.0 \pm 2.8$ & 10.4 \\
\hline
\end{tabular}

Non-REM and REM sleep bout durations were different among the three groups, being significantly shorter in the sleep-deprived rats.

Climbing behavior did not differ among groups on either day (after $24 \mathrm{~h}: \mathrm{F}_{2,21}=0.42, p>0.05$; after $48 \mathrm{~h}: \mathrm{F}_{2,21}=0.42$, $p>0.05$; Figure 1).

\section{Final Conditions}

Plasma corticosterone levels $\left(\mathrm{F}_{2,17}=0.152, p>0.05\right)$ and weight of suprarenal glands $\left(\mathrm{F}_{2,21}=0.261\right)$ were not statistically significant among the groups. Change in body weight induced by the experimental procedure was greater in sleep-deprived and stimulus control animals as compared to the cage control rats $\left(\mathrm{F}_{2,21}=3.965, p<0.05\right.$; Table 3).

\section{DISCUSSION}

In the present report, we have shown that total sleep deprivation, using the disk-over-water method, decreases immobility in the forced-swim test, consistent with many other antidepressant treatment strategies. Thus, this paradigm appears useful for studying the mechanisms underlying the antidepressant effects of sleep deprivation. However, we would like to address possible limitations of the two techniques used (ie, the disk-over-water method of sleep deprivation and the forced-swim test).

As with other methods of sleep deprivation, the major potential confounding factor of the disk-over-water procedure is the stimulation required to maintain forced wakefulness. Forced exercise due to movement of the disk, as well as the possible stress produced by the environment, are extraneous factors that might influence the behavioral outcome. On the other hand, we believe that the effect on the forced-swim test is best explained by sleep deprivation, and not by other factors. In the current protocol, there is a yoked control rat (stimulus control) that received the same degree of stimulation as the paired sleep-deprived rat. Movement of the disk is the same for both rats. In addition, measures of stress, that is, suprarenal weight and plasma 

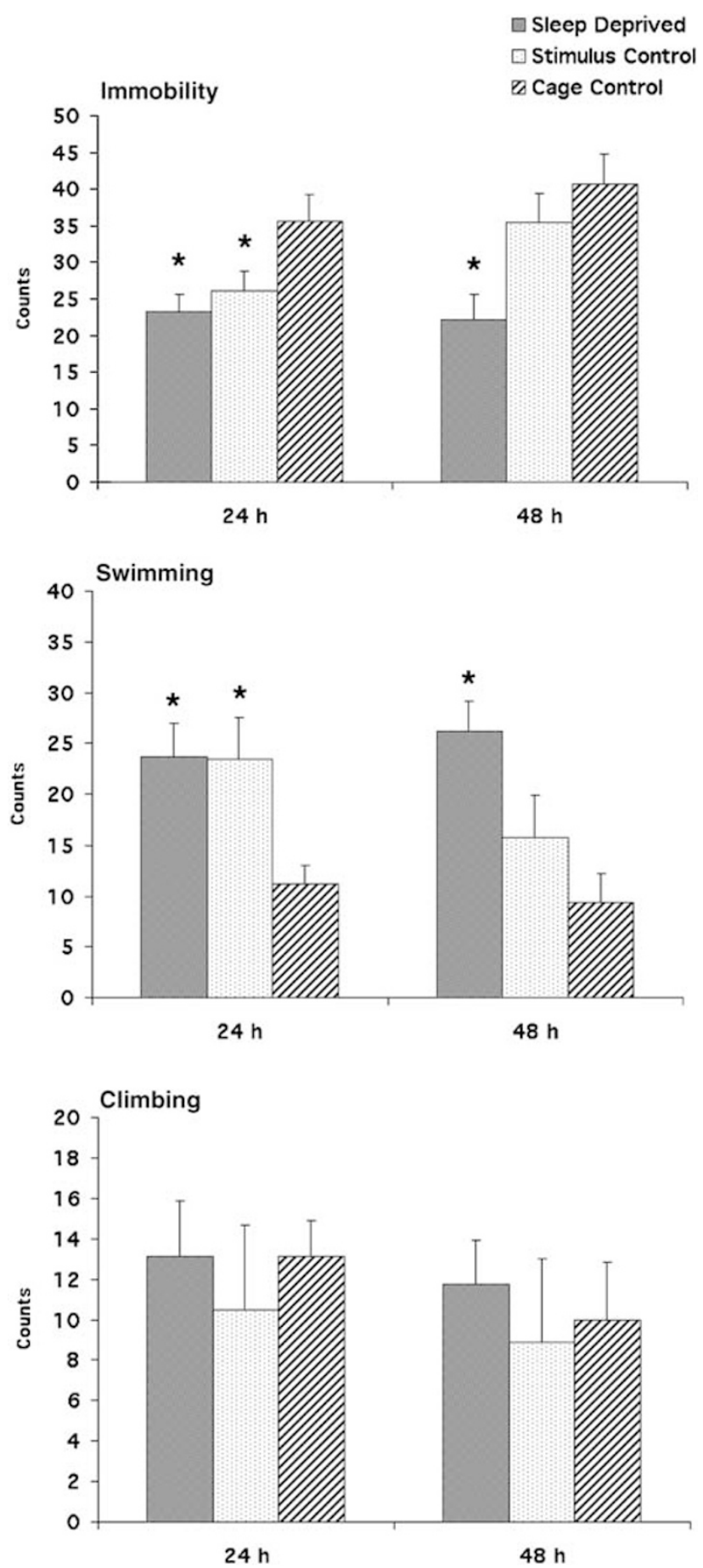

Figure I Changes in activity in the forced-swim test induced by $24,48 \mathrm{~h}$ of sleep deprivation. * Statistically significant difference when compared to cage control. At $24 \mathrm{~h}$ of sleep deprivation increased swimming and decreased immobility when compared to cage control rats. At $48 \mathrm{~h}$ of sleep deprivation increased swimming and decreased immobility when compared to both cage control and stimulus control rats. Histograms represent average \pm SEM.

corticosterone concentrations, also are similar between the two groups of rats, as described previously (Rechtschaffen et al, 1989). On the other hand, the amount of sleep is significantly diminished in the sleep-deprived rats compared to the stimulus-control rats and, hence, sleep
Table 3 Values of Final Conditions After $48 \mathrm{~h}$ of Sleep Deprivation

\begin{tabular}{lccc}
\hline & $\begin{array}{c}\text { Change in total } \\
\text { weight } \mathbf{( g )}\end{array}$ & $\begin{array}{c}\text { Corticosterone } \\
\mathbf{( n g / m l )}\end{array}$ & $\begin{array}{c}\text { Suprarenal } \\
\text { weight }(\mathbf{m g})\end{array}$ \\
\hline Sleep deprived & $29.1 \pm 3.3^{\mathrm{a}}$ & $568 \pm 83$ & $63.8 \pm 2.8$ \\
Stimulus control & $33.1 \pm 4.0^{\mathrm{a}}$ & $538 \pm 55$ & $65.1 \pm 2.6$ \\
Cage control & $9.9 \pm 2.8$ & $518 \pm 56$ & $62.0 \pm 4.2$ \\
\hline
\end{tabular}

${ }^{a}$ Statistically significant differences when compared to cage control. Changes in total weight were significantly different between groups $\left(F_{2,21}=3.965, p<0.05\right)$, being sleep deprived and stimulus control higher than cage control. There was no statistically significant difference among groups in corticosterone levels $\left(F_{2,17}=0.152, p>0.05\right)$ or suprarenal weights $\left(F_{2,21}=0.261, p<0.05\right)$.

deprivation is the most likely cause for the difference in behavior in the forced-swim test between the two groups.

The face validity of the forced-swim test has been questioned because antidepressant drugs require only $24 \mathrm{~h}$ to decrease immobility, which is not consistent with the longer duration of drug treatment needed to induce an improvement in human depression (Willner, 1984). We want to address two points regarding this issue. First, when the dose of the antidepressant is low, chronic administration of the antidepressant is necessary to produce an effect in the forced-swim test, which is congruent with the profile of response in humans (Detke et al, 1997). Second, the neurobiological changes responsible for the decrease in immobility in the forced-swim test after $24 \mathrm{~h}$ of sleep deprivation might model more closely the changes exerted by sleep deprivation in humans to produce antidepressant effects, since a comparable time to produce an effect is required in both species.

Although sleep deprivation exerts a behavioral effect in the forced-swim test after $24 \mathrm{~h}$ when compared with the cage control group, $48 \mathrm{~h}$ are required to obtain differences in immobility with the stimulus control group. A parsimonious explanation for this delay in the differences between sleep-deprived and stimulus control rats resides in the fact that stimulus control rats also are partially sleep deprived. For the first $24 \mathrm{~h}$, stimulus control rats displayed $14 \%$ nonREM sleep, compared with $42 \%$ in the cage control rats. On the second day, stimulus control rats had $25 \%$ non-REM sleep, approaching the levels obtained in cage control. It is possible that this relative increase in non-REM sleep during the second day might be sufficient to block the antidepressant effects exerted by the partial sleep deprivation produced during the first day. This reversal of the effects of sleep deprivation after sufficient sleep also has been described in humans (Gillin et al, 1989), which adds to the predictive validity of the model.

It is unclear whether the behavioral effects of total sleep deprivation are due to deprivation of a distinct stage of sleep. In this regard, deprivation of REM sleep episodes might be an important contributor to the antidepressant effects. Selective REM sleep deprivation in humans also has antidepressant effects, and REM sleep deprivation in animals also induces behavioral activating effects. For example, REM sleep deprivation decreases immobility in the forced-swim test (van Luijtelaar and Coenen, 1985). On the other hand, it is not clear whether deprivation of REM 
sleep is solely responsible for the antidepressant effects of total sleep deprivation. REM sleep deprivation in humans has to continue for several days in order to evoke an antidepressant effect (unlike the $24 \mathrm{~h}$ of total sleep deprivation needed). This suggests that other aspects of sleep probably contribute to the antidepressant effects of total sleep deprivation. Similar findings have been reported in animals using the platform technique of REM sleep deprivation. In rats, van Luijtelaar and Coenen (1985) measured the effect of REM sleep deprivation after $72 \mathrm{~h}$ only, so the effects of shorter REM sleep deprivation are not known. Also, these same authors reported that REM sleep deprivation with the pendulum technique did not modify the behavior in the forced-swim test. Hawkins et al (1980) presented a figure in which the immobility in the REM sleep-deprived rat seems to be different compared to cage control rats after $48 \mathrm{~h}$, although no statistical results for each day of deprivation were provided). However, 4 days were needed to obtain a difference between the sleepdeprived and the stress control rats, unlike the behavioral effects observed herein at $48 \mathrm{~h}$. Thus, we believe that, similar to the findings in humans, total sleep deprivation in rats exerts activating effects more rapidly than REM sleep deprivation.

In the present study, the forced-swim test was performed as described by the Lucki group (Detke et al, 1995). These authors explained that differentiating the behaviors in swimming, climbing, and floating helps to define the neurotransmitter systems involved in the behavioral effect, in that 5-HT antidepressants increase swimming, whereas noradrenergic antidepressants increase climbing (Detke et al, 1995). In our studies, sleep deprivation exerts an effect mainly on swimming behavior, thus resembling the effect of 5-HT antidepressants. In line with this hypothesis, we have previously reported that total sleep deprivation increases extracellular 5-HT levels in the posterior hippocampus of the rat (Lopez-Rodriguez et al, 2003). Additional experiments in which 5-HT synthesis is blocked with p-cholophenylalanine (PCPA) can be performed to test this hypothesis. Involvement of 5-HT in the increased swimming activity was demonstrated by Page et al (1999) by blocking the synthesis of 5-HT with PCPA and suppressing the effects of 5-HT antidepressant drugs.

There is not a well-established explanation as to why sleep deprivation exerts antidepressant effects in humans. Some authors have described that the improvement in depressive symptoms after sleep deprivation in humans is correlated with increased activation (Van Den Burg et al, 1992). Consistent with this hypothesis, various behavioral models also have shown that REM sleep deprivation in rats is associated with behaviors that can be interpreted as increased activity, such as increased exploratory behavior in the open field test, increased swim in the forced-swim test (Hawkins et al, 1980; van Luijtelaar and Coenen, 1985), increased sexual behavior in male rats (Velazquez-Moctezuma et al, 1996), and increased preference for novelty in the y-maze (Moore et al, 1979). Total sleep deprivation also induces increased exploratory behavior in the open field test (Meerlo et al, 1996). It is reasonable to assume that immobility in the forced-swim test might be due to inhibition of activity and that sleep deprivation blocks this inhibition, resulting in an increase in activity. This hypothesis provides construct validity to the paradigm of total sleep deprivation as a fast acting antidepressant strategy with increased activity in the forced-swim test as a behavioral outcome. This is due to the fact that, in spite of the limitations inherent to any animal model of depression (ie, inability to test for subjective experiences), this paradigm models some of the activating effects induced by sleep deprivation in depressed humans, such as improvement in psychomotor retardation (Pflug and Tolle, 1971; Bezzi et al, 1981) and vigor (Szuba et al, 1991). As discussed above, the activating properties might be associated with the antidepressant effect of sleep deprivation (Van Den Burg et al, 1992).

\section{ACKNOWLEDGEMENTS}

We thank Donald Jung for his help with the behavioral scoring of the forced-swim test. Funding was provided by NIMH Grants MH02012 and MH57970.

\section{REFERENCES}

Artigas F, Bel N, Casanovas JM, Romero L (1996). Adaptative changes of the serotonergic system after antidepressant treatments. In: Filippini GA et al (eds). Recent Advances in Tryptophan Research, Vol 398. Plenum Press: New York. pp 51-59.

Bezzi G, Pinelli P, Tosca P (1981). Motor reactivity, pain threshold, effects of sleep deprivation in unipolar depressives. Psychiatr Clin (Basel) 14: 150-160.

Bonnet MH, Arand DL (2003). Clinical effects of sleep fragmentation versus sleep deprivation. Sleep Med Rev 7: 297-310.

Borsini F, Meli A (1988). Is the forced swimming test a suitable model for revealing antidepressant activity? Psychopharmacology 94: $147-160$.

Cryan JF, Lucki I (2000). 5-HT4 receptors do not mediate the antidepressant-like behavioral effects of fluoxetine in a modified forced swim test. Eur J Pharmacol 409: 295-299.

Cryan JF, Markou A, Lucki I (2002). Assessing antidepressant activity in rodents: recent developments and future needs. Trends Pharmacol Sci 23: 238-245.

Detke MJ, Johnson J, Lucki I (1997). Acute and chronic antidepressant drug treatment in the rat forced swimming test model of depression. Exp Clin Psychopharmacol 5: 107-112.

Detke MJ, Rickels M, Lucki I (1995). Active behaviors in the rat forced swimming test differentially produced by serotonergic and noradrenergic antidepressants. Psychopharmacology (Berlin) 121: 66-72.

Gerner RH, Post RM, Gillin JC, Bunney Jr WE (1979). Biological and behavioral effects of one night's sleep deprivation in depressed patients and normals. J Psychiatric Res 15: 21-40.

Gillin JC, Kripke DF, Janowsky DS, Risch SC (1989). Effects of brief naps on mood and sleep in sleep-deprived depressed patients. Psychiatry Res 27: 253-265.

Hawkins J, Phillips N, Moore JD, Gilliland MA, Dunbar S, Hicks RA (1980). Emotionality and REMD: a rat swimming model. Physiol Behav 25: 167-171.

Lopez-Rodriguez F, Wilson CL, Maidment NT, Poland RE, Engel Jr J (2003). Total sleep deprivation increases extracellular serotonin in the rat hippocampus. Neuroscience 121: 523-530.

Meerlo P, Overkamp GJ, Benning MA, Koolhaas JM, Van den Hoofdakker RH (1996). Long-term changes in open field behaviour following a single social defeat in rats can be reversed by sleep deprivation. Physiol Behav 60: 115-119. 
Moore JD, Hayes C, Hicks RA (1979). REM sleep deprivation increases preference for novelty in rats. Physiol Behav 23: 975-976.

Page ME, Detke MJ, Dalvi A, Kirby LG, Lucki I (1999). Serotonergic mediation of the effects of fluoxetine, but not desipramine, in the rat forced swimming test. Psychopharmacology (Berl) 147: 162-167.

Pflug B, Tolle R (1971). Disturbance of the 24-h rhythm in endogenous depression, the treatment of endogenous depression by sleep deprivation. Int Pharmacopsychiatry 6: 187-196.

Pilcher JJ, Huffcutt AI (1996). Effects of sleep deprivation on performance: a meta-analysis. Sleep 19: 318-326.

Poland RE, Lutchmansingh P, Au D, Edelstein M, Lydecker S, Hsieh C et al (1994). Exposure to threshold doses of nicotine in utero: I. Neuroendocrine response to restraint stress in adult male offspring. Life Sci 55: 1567-1575.

Porsolt RD, Anton G, Blavet N, Jalfre M (1978). Behavioural despair in rats: a new model sensitive to antidepressant treatments. Eur J Pharmacol 47: 379-391.

Porsolt RD, Le Pichon M, Jalfre M (1977). Depression: a new animal model sensitive to antidepressant treatments. Nature 266: 730-732.

Post RM, Kotin J, Goodwin FK (1976). Effects of sleep deprivation on mood, central amine metabolism in depressed patients. Arch Gen Psychiatry 33: 627-632.

Rechtschaffen A, Bergmann BM (1995). Sleep deprivation in the rat by the disk-over-water method. Behav Brain Res 69: 55-63.

Rechtschaffen A, Bergmann BM, Everson CA, Kushida CA, Gilliland MA (1989). Sleep deprivation in the rat: X. Integration, discussion of the findings. Sleep 12: 68-87.
Rechtschaffen A, Gilliland MA, Bergmann BM, Winter JB (1983). Physiological correlates of prolonged sleep deprivation in rats. Science 221: 182-184.

Reynolds III CF, Buysse DJ, Kupfer DJ, Hoch CC, Houck PR, Matzzie J et al (1990). Rapid eye movement sleep deprivation as a probe in elderly subjects. Arch Gen Psychiatry 47: 1128-1136.

Szuba MP, Baxter Jr LR, Fairbanks LA, Guze BH, Schwartz JM (1991). Effects of partial sleep deprivation on the diurnal variation of mood, motor activity in major depression. Biol Psychiatry 30: 817-829.

Van Den Burg W, Beersma DG, Bouhuys AL, Van Den Hoofdakker RH (1992). Self-rated arousal concurrent with the antidepressant response to total sleep deprivation of patients with a major depressive disorder: a disinhibition hypothesis. J Sleep Res 1: 211-222.

van Luijtelaar EL, Coenen AM (1985). Paradoxical sleep deprivation, the immobility response in the rat: effects of desipramine, phentolamine. Sleep 8: 49-55.

Velazquez-Moctezuma J, Salazar ED, Retana-Marquez S (1996). Effects of short-, long-term REM sleep deprivation on sexual behavior in male rats. Physiol Behav 59: 277-281.

Vogel GW (1975). A review of REM sleep deprivation. Arch Gen Psychiatry 32: 749-761.

Willner P (1984). The validity of animal models of depression. Psychopharmacology 83: 1-16.

Willner P (1990). Animal models of depression: an overview. Pharmacol Therapeut 45: 425-455. 